\title{
Design of Intensity Modulated Fiber-Optic Temperature Sensor Based on TracePro
}

\author{
Zhihe $\mathrm{Fu}$ \\ Department of Mechanical and Electrical Engineering \\ Longyan College \\ Longyan, China \\ e-mail: 443146226@qq.com \\ Junjie Yang \\ Department of Mechanical and Electrical Engineering \\ Xiamen University \\ Xiamen, China \\ e-mail: 21680188@qq.com
}

\author{
Yibiao Fan \\ Department of Mechanical and Electrical Engineering \\ Longyan College \\ Longyan, China \\ e-mail: 29309975@qq.com \\ Wenxiang Chen \\ Department of Mechanical and Electrical Engineering \\ Xiamen University \\ Xiamen, China \\ e-mail: 646712979@qq.com
}

\begin{abstract}
Compare with traditional way of numerical simulation by establishing the mathematical model through geometry optic, we design a tracepro model to analyze the sensing process of reflective intensity-modulated fiber optic sensor base on ray tracing. We use bimetal as sensing element. This type of sensor has advantages over other fiber optic sensor, including simple structure, flexible design, reliable perform, low cost etc. the linearity and repetition of this sensor were verified during the experiment. The result shows that the maximum error rate of the propone temperature sensor is $3.6 \%$, which appears good accuracy and reproducibility. The measurement system is more sensitive to the vibration test environments, if we can solve this problem, the measurement error is expected to be further improved.

Keywords-Fiber optic sensor;Intensity modulation; Reflective;Temperature; Bimetal;
\end{abstract}

\section{INTRODUCTION}

Reflective intensity-modulated fiber optic sensor (RIM-FOS) are widely used for such physical parameters measurements as distance, vibration, sound, pressure, temperature and acceleration because of their advantages over other fiber sensor, including simple structure, flexible design, reliable perform, low cost etc [1-5]. So far the research of RIM-FOS is based on numerical simulation by establishing the mathematical model through geometry optic. The more complex the reflecting surface is, the more difficult to establish the mathematical model which is suit for qualitative discussions. With the change of the structural parameters, the corresponding mathematical models may need to re-establish. This is unfavorable for application of this type of sensor. In addition in the design process multi-parameter configuration optimization has been a major trouble for designers. To better understand the working principle and improve the performance of the sensor, the ray -tracing simulation was carried out by using optical analysis software TracePro (TP), which provides the sensing process for us.

Meanwhile a RIM-FOS temperature sensor based on bimetal as temperature sensing element is designed [6,7]. Its measurement error and repeatability was verified during the experiments.

\section{TRACEPRO SIMULATION}

A. TP Model

Following assumptions are being considered for the TP model:

- The transmitting fiber (TF) and receiving fiber (RF) have perfectly circular cross sections with radius of $0.1 \mathrm{~mm}$.

- $\mathrm{TF}$ is placed on the focal axis of XY plane.

- Both the fibers are straight parallel, with no space left between them and having the same numerical aperture NA of 0.56 . So the beam divergence angle can be described as

$$
\theta=\sin ^{-1} \frac{N A}{n_{0}}
$$

$n_{0}$ is the refractive index of the medium present in between the fiber tips and the concave reflector.

Here was zero, then $\theta=34^{0}$. A hollow thin round table structure shown in figure 1 was built to compensate the light loss caused by NA, where 
$\mathrm{S} 1$ and S2 plane is set to be completely absorbed in TP.

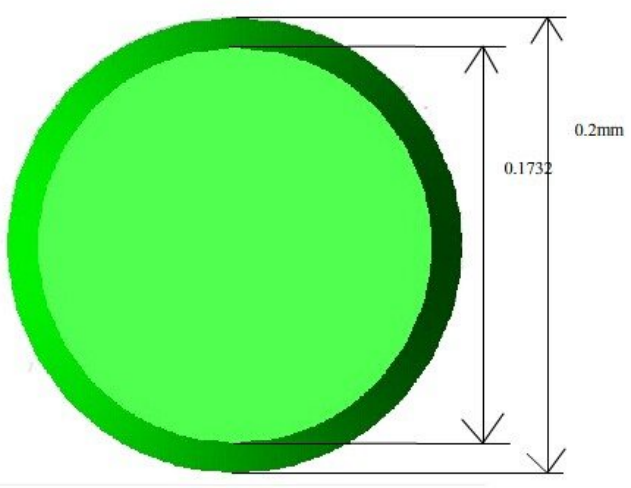

(a)

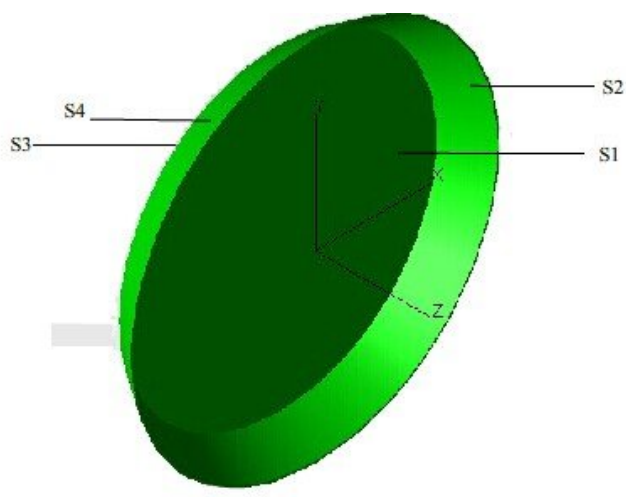

(b)

Figure1. Thin round table structure(a)Bottom view (b) threedimensional view

- Light source: The light emitting characteristics of TF tip was generally recognized as Gaussian distribution [8].For this reason a grid light was set with an outer diameter of $0.1 \mathrm{~mm}$ and divergence angle $34^{\circ}$, the angular distribution was set to be Gaussian density distribution.

\section{B. Verification and Design of the TP Model}

The amount of the light collected by RF is directly correlated to the distance between the fibers tips and the reflector. Therefore, the distance variation can be measured by monitoring the intensity change of the collected light.

Here light intensity function $M$ could be expressed as $[9,10]$

$$
\mathrm{M}=\frac{N}{N_{\text {Tatal }}}
$$

$\mathrm{N}$ represents the light intensity collected from S1 surface, $N_{\text {Total }}$ represent the total light number of light source from TF. In this case coupling efficiency of the light intensity could replace with $\mathrm{M}$.
By changing the distance $d$ which was between reflecting surface center and RF tip (With spacing of $0.02 \mathrm{~mm}$ ) we obtained $\mathrm{M}$ values, the light modulation curve was shown in Figure 2 by Origin software process.

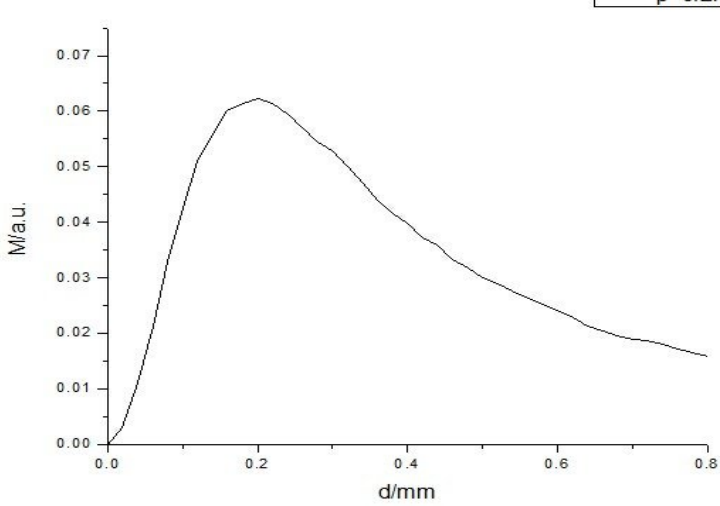

Figure2. Light modulation curve

Figure 2 showed good linearity and sensitivity in $0.02 \sim 0.12 \mathrm{~mm}$.

In order to analyze the distribution of light intensity in the receiving side (XY plane), which is conducive to reasonable arrangements for TF and RF position, a rectangular receiving surface structure $(0.8 * 1.0 \mathrm{~mm})$ was constructed. In the configuration with distance between TF and RF $p=0.2 \mathrm{~mm}$, distance between $\mathrm{TF}$ tip and reflector $\mathrm{d}=0.2 \mathrm{~mm}$, the irradiance of the receiving surface was gain as shown in Fig. 5, the light intensity distribution appear to be the ladder distribution. The cross-sectional view of the right figure shows light intensity distribution of corresponding areas of the horizontal and vertical direction.
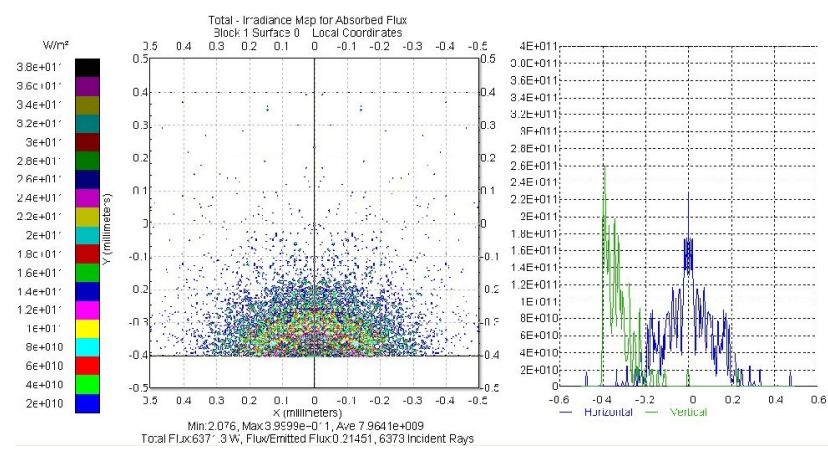

Figure3. Irradiance chart of receiving surface

\section{EXPERIMENT AND ANALYSIS}

Based on the principle above we design experimental apparatus (Figure 4) with $\mathrm{d}=0.02 \mathrm{~mm}$ as starting. The bimetal was used as sensing element. The linearity and repetition of this sensor were verified during the experiment. 
To overcome the effects of the surrounding stray light, we use $38 \mathrm{KHz}$ square wave modulate the emitted light, the measurement system is shown in Figure 5.

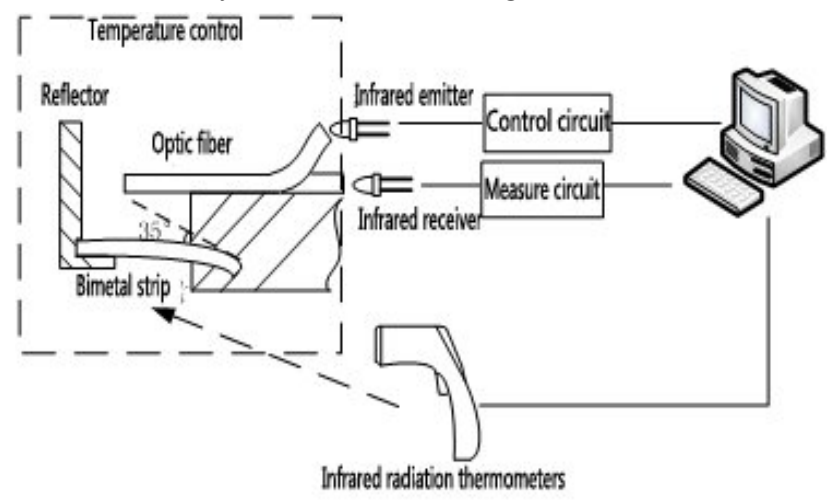

Figure4. Schematic diagram of experimental set-up

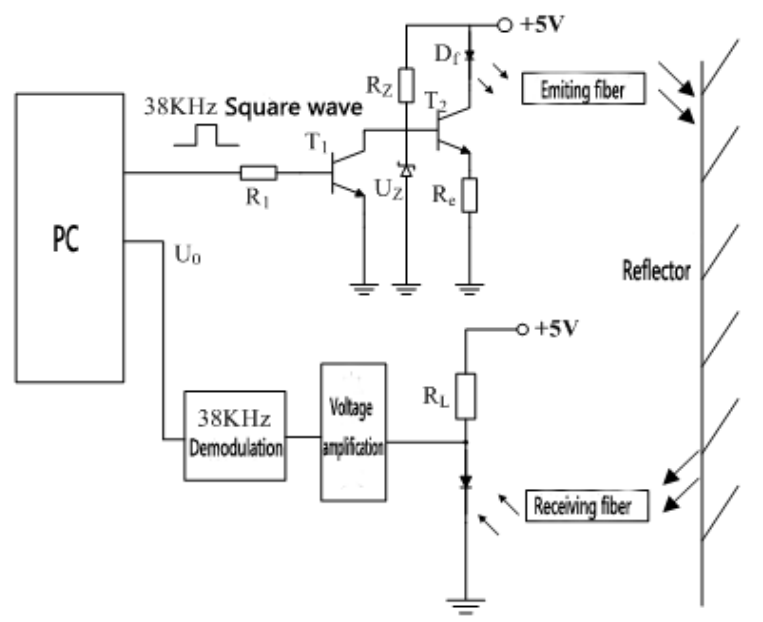

Figure5. Measurement system schematic diagram

As we can see in Figure 5, constant current source circuit which is composed of the emitting portion $\mathrm{R}_{\mathrm{Z}}$ 、 $U_{Z} 、 T_{2} 、 R_{e}$, provide a constant current for infrared emission $\mathrm{D}_{\mathrm{f}}$, ensure $\mathrm{D}_{\mathrm{f}}$ emitted constant intensity infrared light. $\mathrm{T} 1$ is the modulation switch. PC machines emit a square wave with frequency of $38 \mathrm{KHz}$, control constant current source circuit working in $38 \mathrm{KHz}$ square wave by the use of modulation switching .Thus infrared light beam is modulated in the form of pulse light of $38 \mathrm{KHz}$. Receiving portion is composed of infrared photocell, voltage amplifier, $38 \mathrm{KHz}$ demodulator. When the temperature changed, the light signal collected by infrared photocell turn into temperature signal via voltage amplification, demodulation, then transported into PC.

Experiment working on fiber optic temperature sensing system is taken in $30 \sim 120^{\circ} \mathrm{C}$.

As a comparison, we use refit infrared radiation thermometers to measure the temperature. Both the data collected by infrared radiation thermometers and fiber optic temperature measuring equipment are imported into $\mathrm{PC}$ for processing in real time. To control the temperature we use hot air blower motor. While the heating temperature reaches $120{ }^{\circ} \mathrm{C}$ stopped, then temperature was cool naturally. This process is divided into three parts: Fast heating (Fast), step-heating (Step), repeated heating and cooling (Repeated) (Figure 6).This process help to verify that whether or not the relationship between the output voltage of the temperature measuring device and temperature changes is linear and the consistency of repeated measurement results.

Figure 6(a) of shows the experimental curves of fast heating, $0-40$ s to probe pre-heating, $40-80$ s closer to the hot air so fast heating, cooling freedom after 80s; Figure 6 (b) of the experimental curve heating stage $25-40$ s preheated(PH) from room temperature to maintain, $40-85 \mathrm{~s}$ preheating temperature, $85-95 \mathrm{~s}$ rapid heating to $100{ }^{\circ} \mathrm{C}, 95-$ 140 s probe was slowly heated to $120^{\circ} \mathrm{C}$, then free cooling; Figure $6(\mathrm{c})$ is repeated heating and cooling experiment curve, $0-60 \mathrm{~s}, 60-75 \mathrm{~s}$ initial preheating temperature was raised to $120{ }^{\circ} \mathrm{C}, 75-95 \mathrm{~s}$ free cooling of the probe to $70{ }^{\circ} \mathrm{C}, 95-150 \mathrm{~s}$ again heated to $120{ }^{\circ} \mathrm{C}$, free cooling after $150 \mathrm{~s}$.

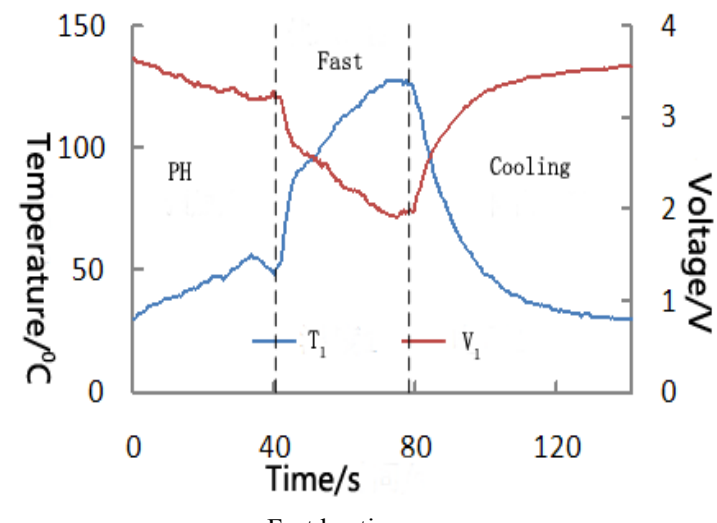

Fast heating

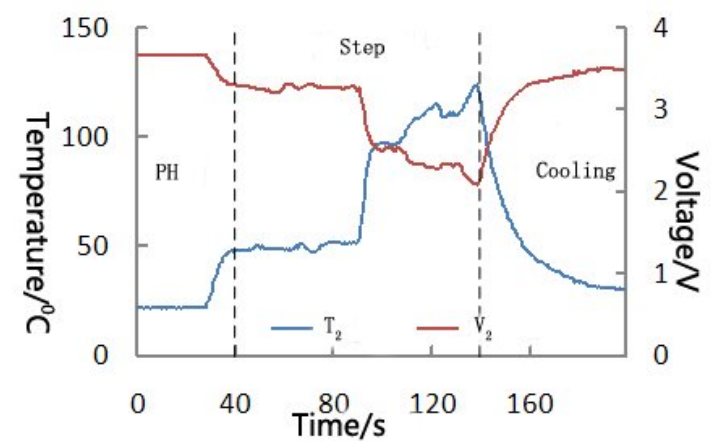

(b) Step heating

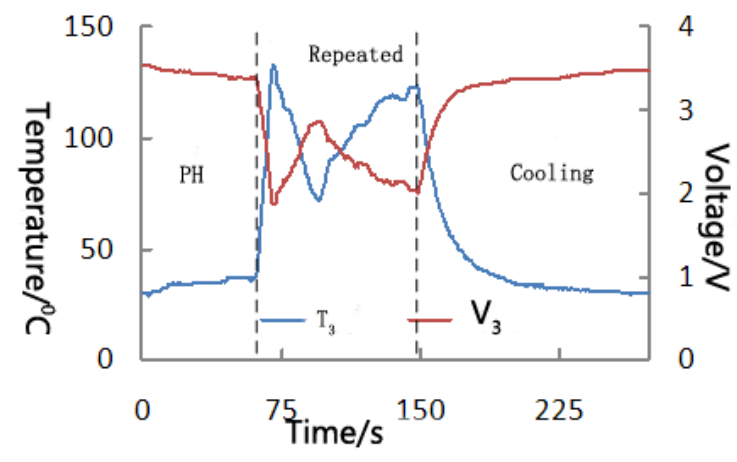

(c) Repeated heating and cooling

Figure6. The curves of voltage and temperature 
Figure 6 shows that this sensor has a good response to temperature, the voltage decreases with increasing temperature, increases while the temperature drop.

To examine whether the relationship between the output voltage and temperature is linear, linear regression analysis is used, Figure 7 shows the measurement results via the data processing. As can be seen, the measured voltage values fluctuate in the vicinity of the regression line, indicating a good linear relationship between voltage and temperature during the heating and cooling process. The fluctuation can be explained as: thermometer errors; random errors cause by fluctuation of hot air blower motor.
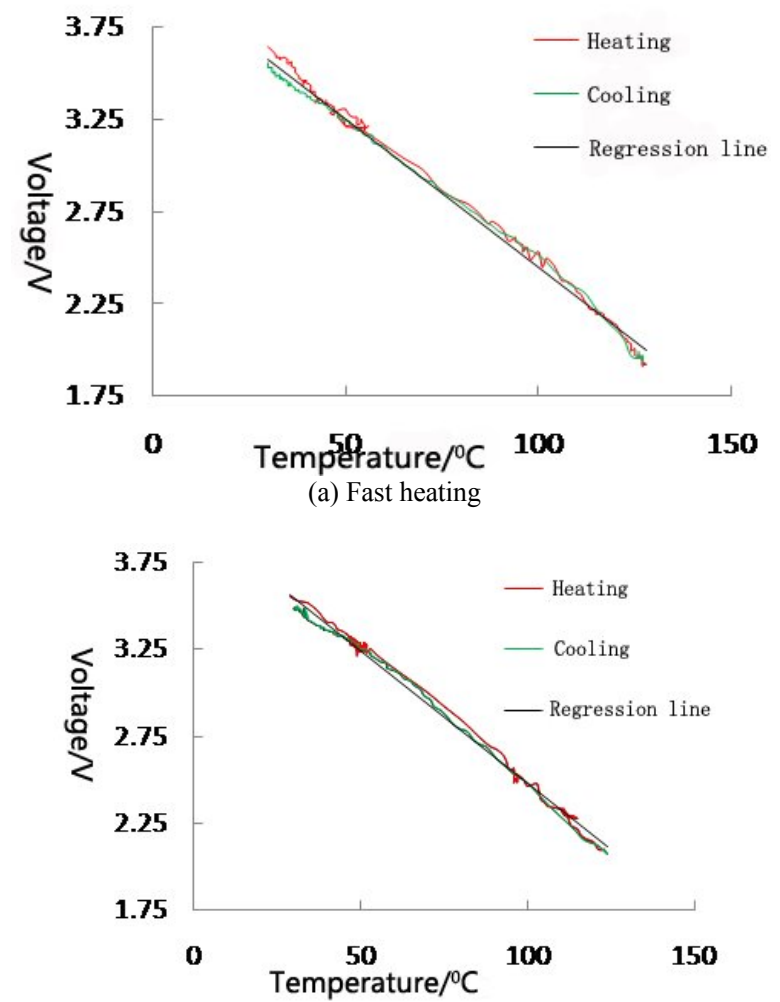

(b) Step heating

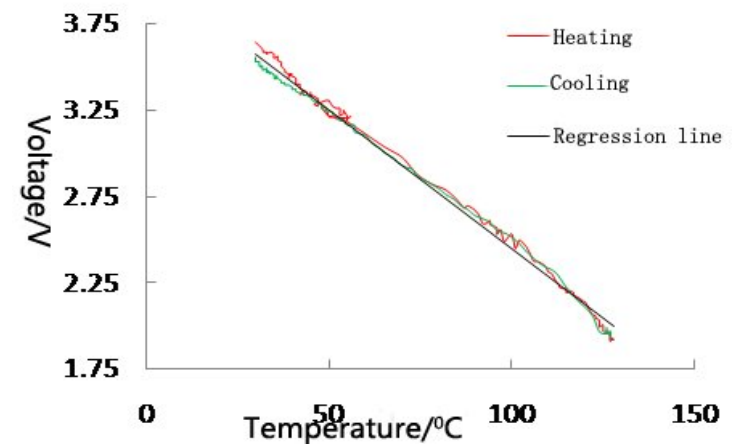

(c) Repeated heating and cooling

Figure1. The curves of voltage and temperature after data processing

In order to analyze the performance of the sensor intuitively, we consider the measurement error and repeatability. Taking into account dense sampling points along the timeline in the experiment, we define the temperature range of the experimental data to facilitate analyzing. To describe the measurement error and repeatability, the maximum error rate $(\mathrm{M})$ and the maximum error rate of repetition $\left(\mathrm{M}_{\mathrm{RP}}\right)$ are defined.

$$
\begin{aligned}
M & =\frac{\operatorname{Max}\left[T_{\text {error }}\right]}{V} \\
\mathrm{M}_{\mathrm{RP}} & =\frac{\operatorname{Max}\left[S-\overline{S_{3}}\right]}{V}
\end{aligned}
$$

Where $T_{\text {error }}$ is the error between the measurement voltage and the point values corresponding to regression line within temperature ranges, $\mathrm{V}$ is the voltage measurement range, $\mathrm{S}$ and $\overline{S_{3}}$ are single regression value and average regression value in three times respectively. $\operatorname{Max}\left[T_{\text {error }}\right]$ can evaluate deviation from the measured data with the regression line, $\mathrm{M}_{\mathrm{RP}}$ can evaluate the repeatability of the measurement process. The results obtained in Table 1 shows that the maximum error rate of the three experiments is $3.6 \%$, which shows good accuracy and reproducibility of the temperature sensor.

TABLEI MAXIMUM ERROR RATE TABLE

\begin{tabular}{|c|c|c|c|c|}
\hline \multirow{2}{*}{ Temperature $\left({ }^{\circ} \mathrm{C}\right)$} & \multicolumn{3}{|c|}{ The maximum error rate $(\%)$} & \\
\cline { 2 - 5 } & Fast & Step & Repeated & Repetitive rate \\
\hline $30 \sim 39$ & 4 & 3 & 4 & 3.6 \\
\hline $40 \sim 49$ & 2 & 2 & 2 & 3.4 \\
\hline $50 \sim 59$ & 3 & 4 & 4 & 3.2 \\
\hline $60 \sim 69$ & 1 & 3 & 3 & 3.0 \\
\hline $70 \sim 79$ & 2 & 4 & 5 & 2.9 \\
\hline $80 \sim 89$ & 3 & 3 & 4 & 2.7 \\
\hline $90 \sim 99$ & 4 & 3 & 4 & 2.5 \\
\hline $100 \sim 109$ & 4 & 2 & 2 & 2.5 \\
\hline $110 \sim 120$ & 3 & 4 & 4 & 2.8 \\
\hline
\end{tabular}

\section{CONCLUSION}

We design a TP model to analyze the sensing process of RIM-FOS. Use bimetal as sensing element, the linearity and repetition of this sensor were verified during the experiment. The result shows that the maximum error rate of the propone temperature sensor is $3.6 \%$, which has good accuracy and reproducibility. The measurement system is more sensitive to the vibration test environments, if we can solve this problem, the measurement error is expected to be further improved.

\section{ACKNOWLEDGMENT}

This work is supported by Cooperation of Industry, Education and Academy from Longyan College (series no. LC2013001).

\section{REFERENCES}

[1] Chengqi Li, Xiaodong Zhang and Ping Zhang, Study on The Compensation Technology for The Intensity Modulated TwoCircle Coaxial Fiber Optic Displacement sensor, in Proceedings of the 2011 IEEE International Conference on Mechatronics and Automation, August 7-10, Beijing, China

[2] P. Puangmali, K. Althoefer, Mathematical modeling of intensitymodulated Bent-tip optical fiber displacement sensors.[J] IEEE Trans. Instrum. Meas. 59, 283-291 (2010) 
[3] P.B. Buchade, A.D. Shaligram, Influence of fiber geometry on the performance of two-fiber displacement sensor.[J] Sens.Actuators A 136, 199-204 (2007)

[4] S. Hadjiloucas, J. Irvine, J. Bowen, Radiometric analysis of the light coupled by optimally cut plastic optical fiber amplitude modulating reflectance displacement sensors. [J] Rev. Sci. Instrum. 71, 3007-3009 (2000)

[5] Praveen Kumar, Shailesh Kumar Singh, Piyush Ojha. Bifurcated Optical Fiber Sensor For Weight Measurement System [C]. International Conference on Computational Intelligence and Communication Systems, 2011,242-245.

[6] LI Y P, GUO X M, et al. Study on Reflection Type Optical Fiber Temperature Sensor [J]. Computer Measurement \& Control, 2010,18 (9) :2201-2202.
LI D K, ZHU X F, et al. Thermal Bimetal Development Review [J]. Shonghai Steel\& Iron Research,1986,1: 50-56.

[7] H.Golnabi Design of an optical fiber sensor for linear thermal expansion measurement [J]. Optics \& Technology,2002, 34:389394.

[8] LI Y F. Study on a New Reflective Optical Fiber Sensor [J]. Chinese Journal of Scientific Instrument, 2005, 26(10):1097-1100.

[9] REN J K. The Research on Output Characteristics of Reflective Intensity-modulated Optical Fiber Displacement Sensors [D].Changsha: Changsha University of Science \& Technology, 2009:11-16.

[10] CHEN Y P, CAO H M, et al. Modeling and Simulation of Reflective Optical Fiber Bundle Displacement Sensors [J]. Journal of Optoelectronics · Laser, 2005,16(6):653-658. 